Michael Darmon Sean M. Bagshaw Lui G. Forni

\section{Balancing the "humors" in severe sepsis: still a role for extracorporeal therapies?}

Received: 2 April 2015

Accepted: 3 April 2015

Published online: 14 May 2015

(C) Springer-Verlag Berlin Heidelberg and ESICM 2015

M. Darmon (『)

Medical-Surgical Intensive Care Unit, Saint-Etienne University Hospital, Avenue Albert Raymond, 42270 Saint-Priest-En-Jarez, France

e-mail: Michael.darmon@chu-st-etienne.fr

Tel.: +33477127853

\section{Darmon}

Jacques Lisfranc Faculty of Medicine, Jean Monnet University, 15 Rue Ambroise Paré, 42023 Saint-Etienne, France

S. M. Bagshaw

Division of Critical Care Medicine, Faculty of Medicine and Dentistry, University of Alberta, Edmonton, Canada

L. G. Forni

Royal Surrey County Hospital NHS Foundation Trust, Surrey Peri-operative Anaesthesia and Critical Care Collaborative Research Group and Faculty of Health and Medical Sciences, University of Guildford, Surrey, UK

\begin{abstract}
Abbreviations
PMX Polymixin hemoperfusion

RCT Randomized controlled trial

RR Relative risk
\end{abstract}

The mortality and morbidity from severe sepsis continue to be a major global health problem. Although the recent data from the Protocol-Based Care for Early Septic Shock
(ProCESS) study showed a lower mortality in severe sepsis than had been demonstrated some 10 years previously, the mortality remains unacceptably high $[1,2]$. The pathogenesis of sepsis is complex, leading to the production of a wide range of inflammatory mediators, which propagate the host response to infection leading to the clinical syndrome of septic shock with multi-organ involvement. At present, treatment relies predominantly on source control, adequate early antimicrobials and organ support where necessary with little effective, targeted therapy [3-5].

It was suggested that extracorporeal blood purification may provide an adjunct for treating severe sepsis by removing harmful inflammatory mediators from the plasma of patients [4]. Furthermore, subsequent improvements with the use of hemofiltration, for example, were reported in both animal and human studies showing that inflammatory cytokines could be removed from the circulation in septic shock $[6,7]$. However, despite early promise, randomised controlled studies have failed to demonstrate any survival benefit from conventional or high volume hemofiltration $[8,9]$. Therapeutic plasma exchange (TPE) has also been used to modulate circulating levels of inflammatory cytokines, coagulation factors and endotoxin; however, despite positive preliminary data, less than 200 patients have been included in trials to date and additional studies are needed before this therapy can be routinely recommended [10]. These therapies are not banal and without potential for adverse effects. For example, standard hemofiltration has been associated with worsening clinical status when applied as adjuncts to the treatment of sepsis [8]. Thus, the risk-benefit ratio of these techniques for many patients remains uncertain. Similarly, the ideal population, context, timing and techniques to derive benefit remain largely unknown. Lastly, the complex balance between pro-inflammatory and anti-inflammatory mediators along with the fact that effects at the cellular level are likely unrelated to circulatory levels are theoretical limits to the effectiveness of blood purification techniques. 
Polymyxin B, a cationic polypeptide antibiotic, displays a high affinity for endotoxin, the lipopolysaccharide complex associated with the outer membrane of Gramnegative pathogens. Endotoxin triggers a signaling cascade for macrophage/endothelial cells to secrete proinflammatory cytokines and nitric oxide, leading eventually to septic shock and multi-organ dysfunction. First described over two decades ago, polymyxin B hemoperfusion (PMX) is widely used in Japan and identified as one of the more promising techniques of blood purification available to date $[10,11]$. A recent systematic review performed on 28 studies (1425 patients) suggested that the use of PMX was associated with improvements in hemodynamic profile, improved lung function, and a measurable reduction in circulation endotoxin levels. Additionally, among the 15 studies in which mortality was reported (920 patients), the pooled mortality rate decreased from 61 to $35 \%$ (RR 0.53, $95 \%$ CI 0.43-0.65) [12]. These promising results were subsequently confirmed by the EUPHAS (early use of polymyxin B hemoperfusion in abdominal septic shock) study, an unblinded randomised trial of PMX hemoperfusion compared to standard care in 64 post-operative patients with an abdominal source of septic shock [13]. The EUPHAS study found that use of PMX hemoperfusion was associated with improved hemodynamic profile and organ dysfunction scores. Furthermore, there was a statistically significant, albeit marginal, decrease in the secondary outcome of day-28 mortality (32 vs. $53 \%$; hazard ratio $0.43,95 \% \mathrm{CI} 0.20-0.94$; RR $0.61,95 \% \mathrm{CI}$ 0.34-1.09) [13]. In contrast, a recent propensity-matched analysis on the effect of post-operative PMX hemoperfusion on mortality in 1180 patients with abdominal septic shock utilizing a large Japanese clinical and administrative inpatient database failed to show a significant improvement in 28-day mortality (17.1\% in the PMX group vs. $16.3 \%$ in matched controls, $p=0.696$ ) [11].

In a recent article in Intensive Care Medicine, the ABDOMIX group reports their findings from the largest randomised controlled study on PMX hemoperfusion in sepsis completed to date [14]. In this trial, patients with documented peritonitis and septic shock were allocated to receive either two sessions of PMX hemoperfusion or usual care following surgery. Allocation was concealed and randomisation was stratified by centre. Despite being unblinded and utilizing a fixed allocation block, the risk of bias seemed relatively limited. The primary endpoint was day-28 mortality and analysis was intention-to-treat. Although only 81 patients ultimately completed both sessions, intention-to-treat and per-protocol analyses found no significant improvement in the primary endpoint of 28-day mortality or any secondary endpoint of interest including improvement in organ dysfunction, hemodynamic profile or mortality at 90 days. In total, among those receiving PMX hemoperfusion, 33 of 119 patients $(27.7 \%)$ died by day 28, while 22 of 113 patients $(19.5 \%)$ died in the standard care group (OR 1.59, $95 \%$ CI 0.86-2.94).

One may now question whether this study casts a shadow on the promise of PMX-based therapy for patients with septic shock. These findings appear discordant with randomised trials, though may not have been entirely unexpected (Fig. 1). For example, in the high profile EUPHAS study, despite improvement in several surrogate endpoints (i.e. hemodynamic profile, organ failure), the trial was not designed to evaluate mortality as a primary endpoint and if any additional patients allocated to the PMX group had died prior to 28 days, there likely would not have been a statistically significant difference found. The ABDOMIX study was undoubtedly a challenging and impressive undertaking; however, it also suffered from methodological limitations that may mitigate the negative result. Firstly, the study was underpowered given the overoptimistic assumptions of both mortality reduction and mortality rate assumptions in the control group, points acknowledged by the authors. Despite this, there was no apparent difference to suggest that PMX treatment improved outcome, implying that PMX hemoperfusion,

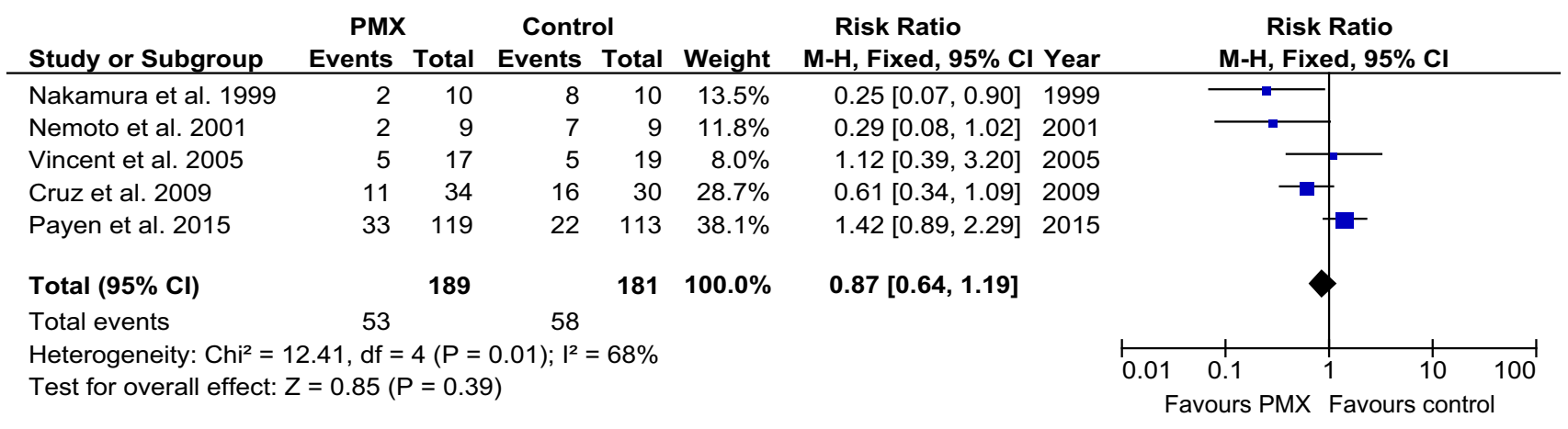

Fig. 1 Summary of the main randomised trials assessing influence of PMX on mortality of patients with severe sepsis or septic shock. Only Payen et al.'s study was considered at low risk of bias [13-17] 
as prescribed in this population, does not likely portend meaningful survival advantage. Secondly, the ABDOMIX trial did not identify patients with the highest likelihood of deriving benefit from PMX therapy by screening patients for elevated circulating levels of endotoxin. This may have inadvertently created misalignment between allocated groups by having a significant proportion of patients randomised to PMX therapy with negative or low endotoxin levels, where the risks of therapy may have outweighed any benefit. Another large multi-centre randomised controlled trial of PMX hemoperfusion in septic shock, the EUPHRATES trial (NCT01046669), aims to enrol 650 patients specifically targeting those with elevated blood endotoxin activity (and therefore those most likely to derive benefit if it exists) and is due for completion by early 2016 . This trial, together with the ABDOMIX trial, will certainly provide clearer insight into the efficacy of PMX therapy and settle existing uncertainties.

Acknowledgments This work was unfunded. Dr. Bagshaw holds a Canada Research Chair in Critical Care Nephrology and is a Clinical Investigator supported by Alberta Innovates-Health Solutions (AI-HS).

Conflicts of interest Dr. Bagshaw is a member of the Steering Committee for the EUPHRATES trial (available at: https://www.clinicaltrials.gov/ct2/show/study/NCT01046669; access date 30 March 2015).

\section{References}

1. Investigators ProCESS, Yealy DM, Kellum JA et al (2014) A randomized trial of protocol-based care for early septic shock. N Engl J Med 370:1683-1693

2. Rivers E, Nguyen B, Havstad S et al (2001) Early goal-directed therapy in the treatment of severe sepsis and septic shock. N Engl J Med 345:1368-1377

3. Parmar A, Langenberg C, Wan L et al (2009) Epidemiology of septic acute kidney injury. Curr Drug Targets 10:1169-1178

4. Dellinger RP, Levy MM, Rhodes A et al (2013) Surviving Sepsis Campaign: international guidelines for management of severe sepsis and septic shock, 2012. Intensive Care Med 39:165-228

5. Levy MM, Rhodes A, Phillips GS et al (2014) Surviving Sepsis Campaign: association between performance metrics and outcomes in a 7.5-year study. Intensive Care Med 40:1623-1633

6. De Vriese AS, Colardyn FA, Philippé JJ et al (1999) Cytokine removal during continuous hemofiltration in septic patients. J Am Soc Nephrol 10:846-853

7. De Vriese AS, Vanholder RC, Pascual $M$ et al (1999) Can inflammatory cytokines be removed efficiently by continuous renal replacement therapies? Intensive Care Med 25:903-910
8. Payen D, Mateo J, Cavaillon JM et al (2009) Impact of continuous venovenous hemofiltration on organ failure during the early phase of severe sepsis: a randomized controlled trial. Crit Care Med 37:803-810

9. Joannes-Boyau O, Honoré PM, Perez P, Bagshaw SM et al (2013) High-volume versus standard-volume haemofiltration for septic shock patients with acute kidney injury (IVOIRE study): a multicentre randomized controlled trial. Intensive Care Med 39:1535-1546

10. Zhou F, Peng Z, Murugan R, Kellum JA (2013) Blood purification and mortality in sepsis: a meta-analysis of randomized trials. Crit Care Med 41:2209-2220

11. Iwagami M, Yasunaga $\mathrm{H}$, Doi $\mathrm{K}$ et al (2014) Postoperative polymyxin B hemoperfusion and mortality in patients with abdominal septic shock: a propensity-matched analysis. Crit Care Med 42:1187-1193

12. Cruz DN, Perazella MA, Bellomo R et al (2007) Effectiveness of polymyxin B-immobilized fiber column in sepsis: a systematic review. Crit Care 11:R47

13. Cruz DN, Antonelli M, Fumagalli R et al (2009) Early use of polymyxin B hemoperfusion in abdominal septic shock: the EUPHAS randomized controlled trial. JAMA 301:2445-2452
14. Payen DM, Guilhot J, Launey Y et al (2015) Early use of polymyxin B hemoperfusion in patients with septic shock due to peritonitis: a multicenter randomized control trial. Intensive Care Med. doi:10.1007/s00134-015-3751-z

15. Nakamura T, Ebihara I, Shoji $\mathrm{H}$ et al (1999) Treatment with polymyxin B-immobilized fiber reduces platelet activation in septic shock patients: decrease in plasma levels of soluble P-selectin, platelet factor 4 and betathromboglobulin. Inflamm Res 48:171-175

16. Nemoto $\mathrm{H}$, Nakamoto $\mathrm{H}$, Okada $\mathrm{H}$ et al (2001) Newly developed immobilized polymyxin B fibers improve the survival of patients with sepsis. Blood Purif 19:361-368 (discussion 368-369)

17. Vincent JL, Laterre PF, Cohen J et al (2005) A pilot-controlled study of a polymyxin B-immobilized hemoperfusion cartridge in patients with severe sepsis secondary to intraabdominal infection. Shock 23:400-405 